Department of Neuroscience, The Ohio State University and the Comprehensive Cancer Center, Columbus, Ohio 43210, USA

M C Früwald

Department of Veterinary Biosciences, The Ohio State University and the Comprehensive Cancer Center, Columbus, Ohio 43210, USA

L J Rush

Department of Biochemistry and Molecular Biology, Mayo Clinic, Rochester, Minnesota 55905, USA

J L Reiter

Department of Pathology, The Ohio State University and the Comprehensive Cancer Center, Columbus, Ohio 43210, USA

G Wenger

University of California, San Francisco, CA 94115-0128, USA J F Costello

Division of Oncology, The Children's Hospital of Philadelphia and the University of Pennsylvania, Philadelphia, Pennsylvania 19104-4318, USA

P S White

G M Brodeur

Continued overleaf

\title{
Gene amplification in PNETs/medulloblastomas: mapping of a novel amplified gene within the $M Y C N$ amplicon
}

Michael C Frühwald, M Sue O’Dorisio, Laura J Rush, Jill L Reiter, Dominic J Smiraglia, Gail Wenger, Joseph F Costello, Peter S White, Ralf Krahe, Garrett M Brodeur, Christoph Plass Abstract
Objectives-The pathological entity of
primitive neuroectodermal tumour/
medulloblastoma (PNET/MB) comprises
a very heterogeneous group of neoplasms
on a clinical as well as on a molecular
level. We evaluated the importance of (PNETs) of the CNS. scanning (RLGS), a method that allows the detection of low level amplification, was used. RLGS provides direct access to DNA sequences circumventing positional cloning efforts. Furthermore, we analysed several samples by CGH. Design-Twenty primary medulloblastomas, five supratentorial PNETs, and five medulloblastoma cell lines were studied. Results-Although our analysis confirms that gene amplification is generally a rare event in childhood PNET/MB, we found a total of 17 DNA fragments that were amplified in seven different tumours. Cloning and sequencing of several of these fragments confirmed the previous finding of MYC amplification in the cell line D341 Med and identified novel DNA sequences amplified in PNET/MB. We describe for the first time amplification of the novel gene, NAG, in a subset of PNET/MB. Despite genomic amplification, $N A G$ was not overexpressed in the tumours studied. We have determined that $N A G$ maps less than $50 \mathrm{~kb} 5^{\prime}$ of $D D X 1$ and approximately $400 \mathrm{~kb}$ telomeric of MYCN on chromosome 2 p24. Conclusion-We found a similar but slightly higher frequency of amplification than previously reported. We present several DNA fragments that may belong to the CpG islands of novel genes amplified in a small subset of PNET/MB. As an example we describe for the first time the amplification of $N A G$ in the $M Y C N$ amplicon in PNET/MB. (F Med Genet 2000;37:501-509)

Keywords: medulloblastoma; PNET; $N A G$ amplification; RLGS

Recent studies have attempted a molecular staging of childhood PNET/MB. Loss of heterozygosity $(\mathrm{LOH})$ for chromosome $17 \mathrm{p}$ is present in up to $50 \%$ of medulloblastomas (infratentorial PNETs), while it was not observed in supratentorial PNETs. ${ }^{5}{ }^{6}$ In addition, there is an association between $\mathrm{LOH}$ for $17 \mathrm{p}$ and metastatic disease and a strong correlation between $M Y C$ amplification and poor prognosis in patients with PNET/MB. ${ }^{7}$ The amplification of proto-oncogenes such as $M Y C$ has been used as a marker for advanced stage disease in other childhood tumours, especially neuroblastoma. A direct correlation between copy number in the proto-oncogene MYCN and clinical outcome is used to assign patients with neuroblastomas to therapeutic groups. $^{8}$

Gene amplification appears to be an uncommon event in childhood PNET/MB. Fuller et $a l^{9}$ pooled data from five independent studies and found that amplification of the genes for $M Y C, M Y C N$, and the epidermal growth factor receptor $(E G F R)$ together was present in only $10 \%$ of medulloblastomas. DNA amplification often results in increased expression of otherwise tightly controlled genes. However, alternative mechanisms that augment gene expression exist. ${ }^{10-12}$ Amplification of certain genes was originally described as a mechanism to prevent drug toxicity; it is now clear that gene amplification plays an important role in the progression of neoplasias owing to overexpression of growth promoting oncogenes. ${ }^{12}$ The amplicon size in tumour cells is usually much bigger than the transcriptional unit of a single oncogene and includes several coamplified genes, a situation which complicates the identification of the actual target gene. $M Y C N$ amplicon sizes range from $350 \mathrm{~kb}$ to more than $1 \mathrm{Mb}{ }^{13}{ }^{14}$ It has therefore been suggested that genes other than $M Y C N$ within the associated amplicon may contribute to the oncogenic potential of this amplification. ${ }^{15} 16$

Previous studies have shown the utility of restriction landmark genomic scanning (RLGS) in the analysis of DNA amplification in human tumour samples ${ }^{17-19}$ because the intensities of DNA fragments visualised on RLGS autoradiographs correlate accurately with DNA copy number. Therefore, RLGS can be used to detect even low levels of gene amplification.

In the current study we used RLGS to evaluate the importance of DNA amplification in PNET/MB of the CNS. DNA fragments showing enhanced intensity on RLGS profiles were cloned by means of a new and efficient technique. ${ }^{17}$ Our analysis showed that gene 
amplification is an underestimated event in PNET/MB. We provide the first data on amplification and chromosomal mapping of the gene $N A G$, coamplified in the $M Y C N$ amplicon in supratentorial PNETs and medulloblastomas.

\section{Methods}

PATIENTS AND CELL LINES

Twenty five PNET/MB samples were analysed (table 1). All samples were obtained through the Cooperative Human Tissue Network (CHTN, Midwestern Division) in accordance with NIH guidelines. Tumour tissue was obtained at neurosurgery and immediately frozen in liquid nitrogen. Control DNA was from normal cerebella ( 4 year old female, 4 day old male, 11 year old male), an adult cortex, and several peripheral blood lymphocyte (PBL) samples (newborn, 3-4 years, 8-9 years, and adult). Control tissues were derived from healthy donors or patients that had died of causes unrelated to neoplasia or neurological disease. Procedures were approved by the Children's Hospital's review board. The cell lines Daoy, D283 Med, and D341 Med were obtained from ATCC (Rockville, MD) and the lines MHH-MED-1 and MHH-PNET-5 were provided by T Pietsch (Universität Bonn, Germany). Cells were cultured under conditions previously published with the exception that human umbilical cord blood serum was used as a supplement. ${ }^{20}$

\section{ISOLATION OF NUCLEIC ACIDS}

High molecular weight DNA for the RLGS procedure was isolated according to our previously published protocol. ${ }^{17}$ Plasmid DNA was isolated using a QIAGEN Plasmid Mini kit (Qiagen Inc, Santa Clara, CA) and recommended protocols. Total RNA was isolated using the TRIzol reagent (GIBCO, Grand Island, NY) according to the distributor's suggestions.

RLGS

RLGS analyses of tumours and control DNA were performed as previously described. ${ }^{21}$ All tumour and cell line RLGS profiles were compared to control profiles from three normal cerebella, an adult cerebral cortex, and at least four peripheral blood lymphocyte (PBL) profiles from children of different age groups. RLGS profiles were analysed by overlaying tumour with normal profiles and marking differences in fragment intensity on clear acetate sheets. Fragment intensity was scored by comparing individual to surrounding single copy fragments. Amplification was estimated according to the difference in intensity. To obtain information on the chromosomal location of the fragments of interest, profiles were compared to an overlay taken from a chromosome assigned RLGS (CA-RLGS) profile created by Yoshikawa et al. ${ }^{22}$

CLONE ISOLATION AND RESTRICTION MAPPING $N o t \mathrm{I} / E c o \mathrm{RV}$ clones were isolated from a human genomic library previously established in our laboratory. ${ }^{23}$ Clones of interest were identified using a catalogue of RLGS mixing profiles. The construction and applications of this tool have recently been described. ${ }^{17}$

SOUTHERN ANALYSIS AND PROBES

To confirm amplification of the DNA fragments 4C20 and 4C23, genomic DNA was digested with $E c o$ RV alone and in combination with NotI. A total of $7.5 \mu \mathrm{g}$ DNA were separated in $0.8 \%$ agarose gels and transferred onto nylon membranes by vacuum blotting (Pharmacia). Probes for hybridisation included the $1.3 \mathrm{~kb} N o t \mathrm{I} / E c o$ RV clone $4 \mathrm{C} 20$, a $600 \mathrm{bp}$ $E c o R V / S a c I$ fragment of $4 \mathrm{C} 23$, and a $400 \mathrm{bp}$ $\mathrm{Xba \textrm {I }}$ fragment from exon 2 of $M Y C N$.

CHROMOSOMAL MAPPING

Human specific PCR primer pairs of clones $4 \mathrm{C} 20$ and 4C23 were designed from the corresponding Not $\mathrm{I} / E \mathrm{C} o \mathrm{RV}$ clones. The primers were: 4C20-F, 5'-GGC AAC AAA CAT GGT GC-3' and 4C20-R, 5'-TCC ATC AGG GAG CGA AAA GAG-3'; 4C23-1, 5'-GGG GAG CAG GTT ATC TGT CAT-3' and 4C23-2, 5'-AGTAGG CAG AAG TAC AGG GC-3'; and 4C23-3, 5'-AGA GGA CAA GGA GCA GGA CAA GC-3' and 4C23-4, 5'-GGG GAC AGA ACC AAG AAT CA-3' amplifying fragments of 115 bp, 136 bp, and 143 bp, respectively. PCR conditions were initial denaturation at $95^{\circ} \mathrm{C}$ for five minutes followed by $30-35$ cycles of $95^{\circ} \mathrm{C}$ for 30 seconds, $59^{\circ} \mathrm{C}$ for 30 seconds, and $72^{\circ} \mathrm{C}$ for 30 seconds, and a final extension of $72^{\circ} \mathrm{C}$ for 10 minutes. Initial chromosomal assignment was performed with a mono-chromosomal human-rodent somatic cell hybrid panel as described elsewhere. ${ }^{24}$ For regional assignment and fine mapping, the GeneBridge 4 (GB4RH), ${ }^{25}{ }^{26} \mathrm{G} 3,{ }^{27}$ and TNG4 (http://wwwshgc.stanford.edu/RH/) radiation hybrid mapping panels were used. ${ }^{28}$ Minimum lod score thresholds of 15 and 4 (as the chromosomal assignment could be specified), respectively, for linkage to markers in the framework map were used for analysis of the GB4 and G3/TNG mapping panel data as recommended in the instructions for the respective $\mathrm{RH}$ mapping servers (http://carbon.wi.mit.edu:8000/cgi-bin/ contig/rhmapper.pl and http://www-shgc. stanford.edu/RH/rhserverformnew.html). The cytogenetic location of each clone was obtained by determining the location of the flanking framework markers in the location database map (http://www.cedar.soton.ac.uk/). Each RH mapping analysis was done in duplicate.

COMPARATIVE GENOME HYBRIDISATION CGH was performed according to the method described by Kallioniemi et al. ${ }^{29}$

MAPPING OF NAG TO A MYCN YAC CONTIG

Clones for a yeast artificial chromosome (YAC) contig of the MYCN locus have been described previously. ${ }^{30}$ Yeast colonies were picked from AHC plates, dissolved in $50 \mu \mathrm{H}_{2} \mathrm{O}$, and then heated in a thermal cycler to $94^{\circ} \mathrm{C}$ and $55^{\circ} \mathrm{C}$, each for five minutes in three sequential steps. 


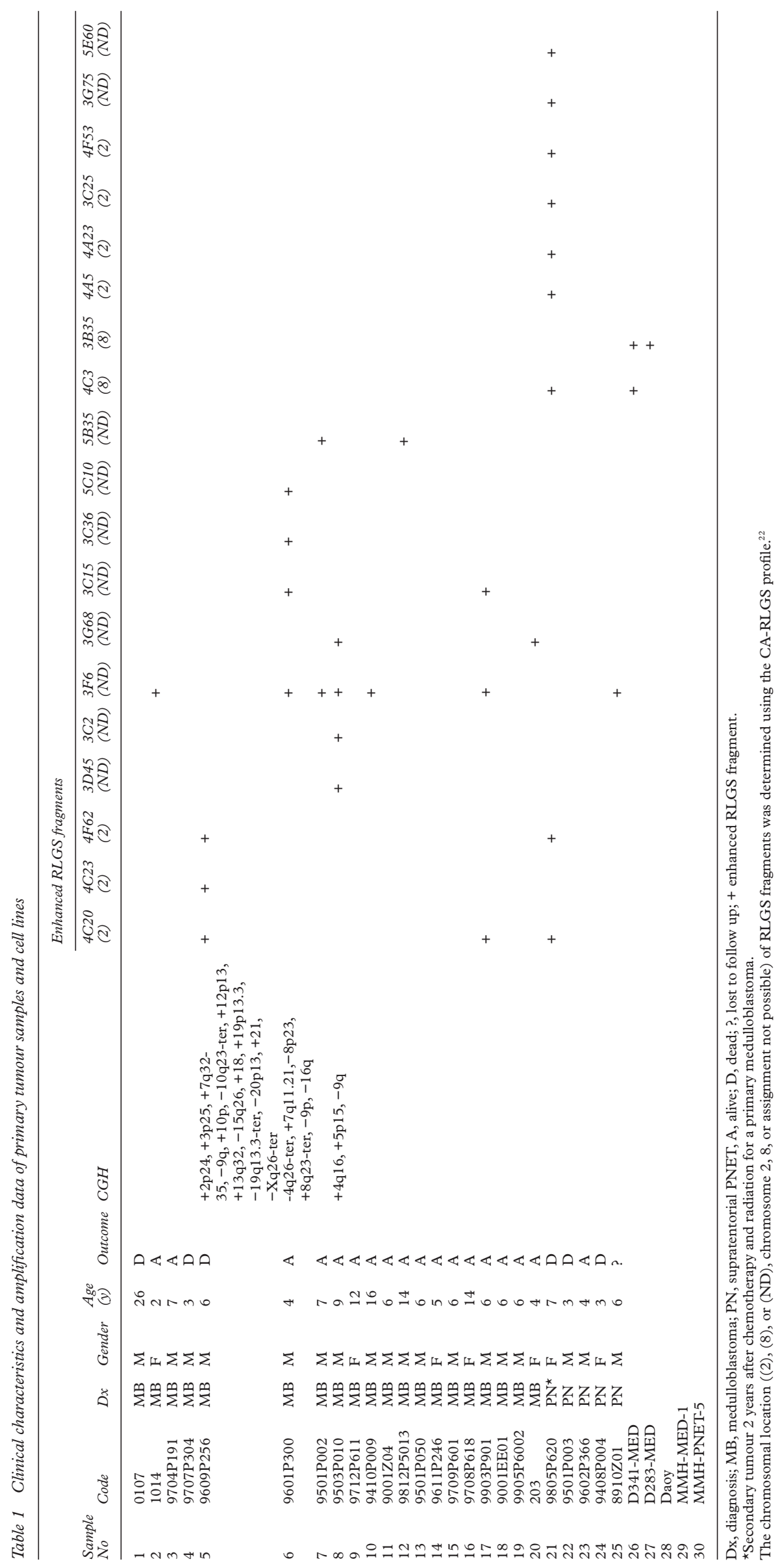


The solution was spun down and $5 \mu$ of the supernatant were used for PCR. To confirm the results of the PCR, 200-500 ng of YAC DNA were digested with EcoRI and electrophoresed on $0.8 \%$ agarose gels and then Southern blotted and hybridised with a probe derived from RT-PCR amplification of EST $k 6533$. The primers used for this were: forward 5'-GAG GCA ACC AAA AAC ATG GT-3' and reverse 5'-TGC CTG GAA GGA CCA AAT CC-3'. The resulting PCR product was $170 \mathrm{bp}$.

SEQUENCING AND DATABASE ANALYSIS

All sequence analyses were performed in the Core Facility of the Division of Human Cancer Genetics using an ABI PRISM 377 DNA sequencer. For CG rich sequences, high annealing temperatures were used with an $\mathrm{ABI}$ PRISM BigDye Terminator Cycle Sequencing kit. NotI/EcoRV clones were sequenced with M13 forward and reverse oligonucleotides and by primer walking. DNA sequence files were analysed using DNAstar and Chromas software. For homology searches, sequences were submitted to the publicly available databases.

SEMIQUANTITATIVE RT-PCR

Five $\mu \mathrm{g}$ of total RNA were reverse transcribed in a volume of $20 \mu \mathrm{l}$ for each primer, using Thermoscript (Gibco BRL, Grand Island, NY) and oligo-dT as well as random hexamers according to the manufacturer's recommendations. After termination of the transcription reaction, oligo-dT, and random hexamer primed cDNAs were combined and diluted to $80 \mu \mathrm{l}$. One $\mu \mathrm{l}$ of the resulting mix was used for RT-PCR experiments. Primers for the housekeeping gene, GPI (glucose phosphate isomerase), were GPIf 5'-GAC CCC CAG TTC CAG AAG CTG C-3' and GPIr 5'-GCA TCA CGT CCT CCG TCA CC-3' (178 bp). Primers for k6533 were k6533F 5'-AGC TTG GCC ACC TAT GAA AA-3' and k6533R 5'-AGG GAC TGG TTA AGC AGC AG-3' (210 bp). All RT-PCR primer pairs spanned introns. Forward primers were end labelled with $\left[{ }^{32} \mathrm{P}\right] \mathrm{dATP}$ using $\mathrm{T} 4$ polynucleotide kinase (Gibco BRL). Multiplex PCR reactions were carried out in $50 \mu \mathrm{l}$ using $1 \mu \mathrm{l}$ cDNA, $10 \mathrm{pmol}$ of each primer (1:10 labelled/unlabelled), 1.5 $\mathrm{mmol} / 1 \mathrm{MgCl}_{2}, 0.2 \mathrm{mmol} / 1$ each dNTP, $10 \times$ PCR reaction buffer (Gibco BRL), $2.5 \%$ DMSO (dimethylsulphoxide), and 2.5 U Platinum Taq (Gibco BRL). Reactions were performed in a Perkin Elmer 9700 GeneAmp thermal cycler. Initial denaturation was $95^{\circ} \mathrm{C}$ for 10 minutes, followed by 21 cycles of $96^{\circ} \mathrm{C}$ for 30 seconds, $58^{\circ} \mathrm{C}$ for 30 seconds, and $72^{\circ} \mathrm{C}$ for one minute. Twenty one cycles were determined to be within the exponential range of amplification for both GPI and k6533 by ImageQuant software (Molecular Dynamics, Sunnyvale, CA). Eight $\mu$ l of each reaction were run on an $8 \%$ polyacrylamide gel and bands were visualised using a phosphorimager (Molecular Dynamics). The ratio of GPI to k6533 products was determined for each sample using the ImageQuant software. All experiments were performed in duplicate.

NORTHERN HYBRIDISATION

Hybridisation of multi-tissue northern blots, MTN-Blots, (Clonetech, Palo Alto, CA) was performed as recommended by the supplier.

\section{Results}

DETECTION OF ENHANCED DNA FRAGMENTS IN PNET/MEDULLOBLASTOMA BY RLGS

Twenty medulloblastomas and five supratentorial PNETs were analysed by RLGS for the presence of DNA amplification. RLGS profiles were created using NotI as the restriction landmark enzyme followed by EcoRV and HinfI digests. Tumour samples were scored for the presence of enhanced RLGS fragments indicative of DNA amplification. A total of 19 different RLGS fragments showed a higher intensity in the tumour profiles compared to control profiles (fig 1). A summary of the tumours that exhibited enhanced fragment intensities and the putative chromosomal location of the corresponding DNA fragments as far as we could evaluate from chromosome assigned RLGS (CA-RLGS) is provided in table $1 .^{22}$

Because Not $\mathrm{I}$ is a methylation sensitive restriction enzyme, the analysis of DNA amplification is complicated. Enhanced fragments in a tumour profile may result from either DNA amplification or hypomethylation of normally methylated NotI sites. Indeed, two of the 19 enhanced fragments (3F6 and 3G68) that we observed had been identified previously as repetitive elements, which exhibit variable hypomethylation. ${ }^{31} 32$

Equal enhanced fragment intensities on the same RLGS profile suggest that fragments belong to the same amplicon. This notion is supported by the finding that the fragments 4C20, 4C23, 4F62, 4A5, 4A23, 3C25, and $4 \mathrm{~F} 53$ are all located on chromosome 2 , as determined by comparison of RLGS profiles with the CA-RLGS profile by Yoshikawa et al. ${ }^{22}$ In addition, fragments $4 \mathrm{C} 20$ and $4 \mathrm{C} 23$ detect the same sized amplified fragment on Southern analysis in tumour sample No 5 (data not shown). Tumour sample No 21 showed two groups of enhanced DNA fragments: group 1, 4C20, 4F62, 4A5, 4A23, 3C25, 4F53 presumably all located on chromosome 2 and group 2 , $4 \mathrm{C} 3$ and 3B35 localised to chromosome 8 as described above. The DNA fragments within a group exhibited equal intensity on autoradiography, while there was a distinct difference in intensity between fragments of groups 1 and 2 suggesting their derivation from two different amplica.

Furthermore, three samples (Nos 5, 6, and 8) that had shown fragment enhancements on RLGS were analysed using CGH (table 1). Among other cytogenetic aberrations medulloblastoma sample No 5 showed an amplification of $2 \mathrm{p} 24$. Tumour sample No 8 showed, besides other rearrangements, gain on the short arm of chromosome 5 ( $5 \mathrm{p} 15)$ a region that has been found amplified in medulloblastomas. ${ }^{33}$ RLGS fragments 3D 45 or 3C2 could be derived from this amplicon on $5 \mathrm{p} 15$. Sample 
No 6 exhibited gains on chromosome $7 \mathrm{q}$ and $8 \mathrm{q}$ and RLGS fragments 3C15, 3C36, or $5 \mathrm{C} 10$ could represent these amplica.

Excluding the two demethylated repetitive element sequences described above, a total of 17 DNA fragments were found enhanced in seven out of $25 \mathrm{PNET} / \mathrm{MB}$. One recurrent PNET (No 21) showed the maximum of eight aberrant DNA fragments, supporting the fact that genetic abnormalities, including gene amplifications, accumulate in the course of disease progression.

In summary, we have identified three tumours (Nos 5, 17, and 21) with amplification involving a region on chromosome $2 \mathrm{p} 24$, possibly $M Y C N$, one tumour (No 8) with possible amplification of $4 q 16$ or $5 \mathrm{p} 15$, and a fifth tumour (No 6) with gains on $7 \mathrm{q} 11$ and $8 \mathrm{q} 23$. Two additional tumours (Nos 7 and 12) showed enhancement of DNA fragment 5B35, which could either represent DNA amplification or hypomethylation since both tumours showed enhancement of the previously described hypomethylated fragment 3F6 (table $1)$. Thus a minimum of five out of $25(20 \%)$ tumours exhibited gene amplification, confirmed by at least two different techniques (CGH and RLGS or RLGS and Southern blotting). This number is higher than the $10 \%$ published and could be as high as $28 \%$ (seven tumours with enhanced RLGS fragments out of a total of 25 analysed tumours). ${ }^{9}$
INCREASED FRAGMENT INTENSITY CORRESPONDS TO DNA AMPLIFICATION

To confirm the results of our RLGS analysis, the enhanced DNA fragments 4C20, 4C23, and 3B35 were cloned and sequenced. ${ }^{17}$ Fragment 3B35 was mapped to chromosome 8 by comparison to the CA-RLGS profile and identified as part of the $5^{\prime} \mathrm{CpG}$ island and first exon of the $M Y C$ gene. ${ }^{17}$ This fragment was enhanced only in the cell lines D283 Med and D341 Med. We estimated a 20-fold amplification in D341 Med and a five-fold amplification in the D283 Med RLGS profile. This is in accordance with the data previously published. $^{34} 35$

Chromosomal assignment of 4C20 and 4C23 using a mono-chromosomal mapping panel verified the localisation obtained by comparison to the CA-RLGS profile to chromosome 2. When used as a probe for Southern analysis on primary medulloblastomas and supratentorial PNETs, 4C20 detected amplification of this DNA in the same samples as seen on RLGS (Nos 5, 17, and 21). Fig 2A shows as a representative example the Southern blot of tumour No 21. The same level of amplification as for $4 \mathrm{C} 20$ was seen for $4 \mathrm{C} 23$, which was changed only in tumour sample No 5 (data not shown). To refine the chromosomal location, we carried out radiation hybrid mapping with three independent RH mapping panels providing increasingly higher levels of resolution.
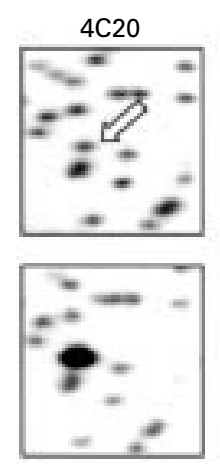

$9805 \mathrm{P} 620$
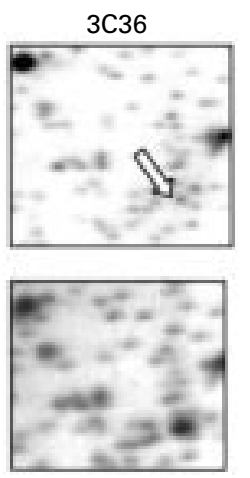

9601P300
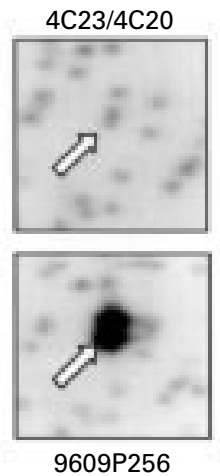

9609P256
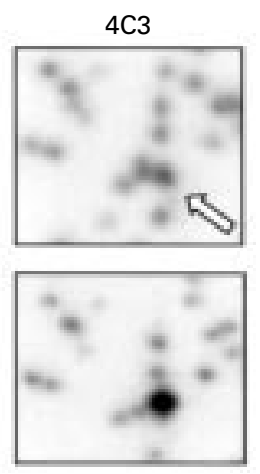

9805P620
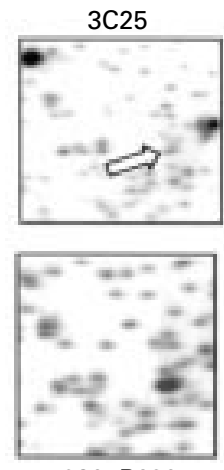

9805P620
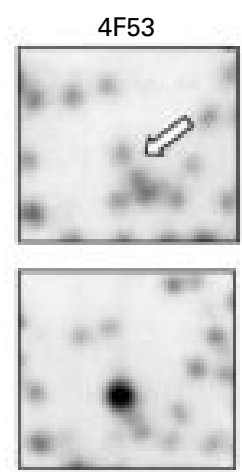

9805P 620
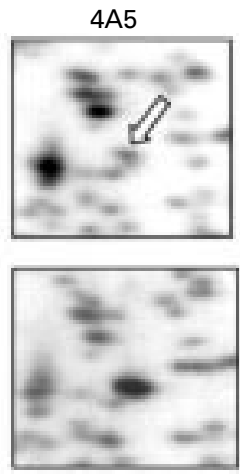

9805P620
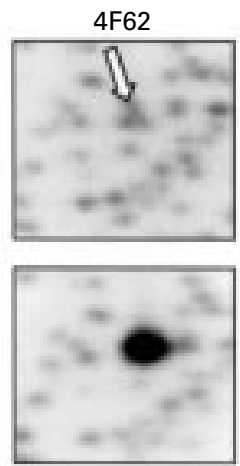

9609P256

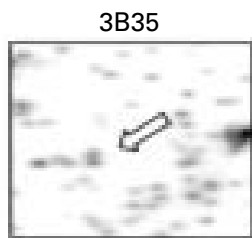

Cerebellum

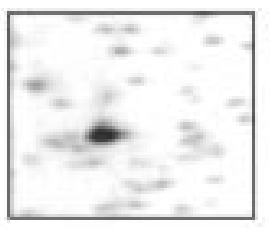

Tumour

D341Med
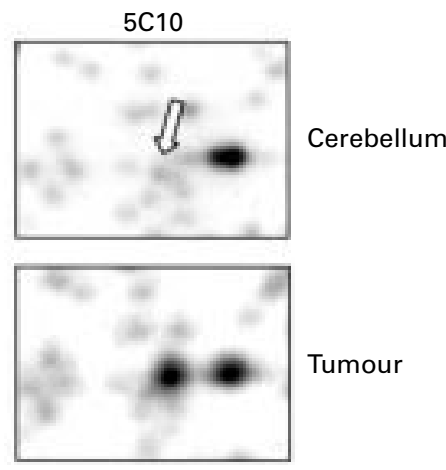

$9601 \mathrm{P} 300$

Figure 1 Detection of enhanced DNA fragments in PNET/MB on RLGS profiles. Shown is a comparison between DNA fragments, which are enhanced on RLGS profiles from PNET/MB, with single copy fragments in normal cerebellum profiles. Open arrows point to single copy fragments in the normal cerebellum profile (top rows) that are amplified in the tumours (bottom rows). The arrow points to the enhanced RLGS fragment $4 C 23$ of tumour sample No 5 (9609P256). The number on top of each pair of RLGS profile sections is the RLGS DNA fragment identifier. The number on the bottom of each pair identifies the tumour samples. All normal RLGS profile sections in this figure were taken from the cerebellum profile of a 4 year old male who had died from a cardiomyopathy. 

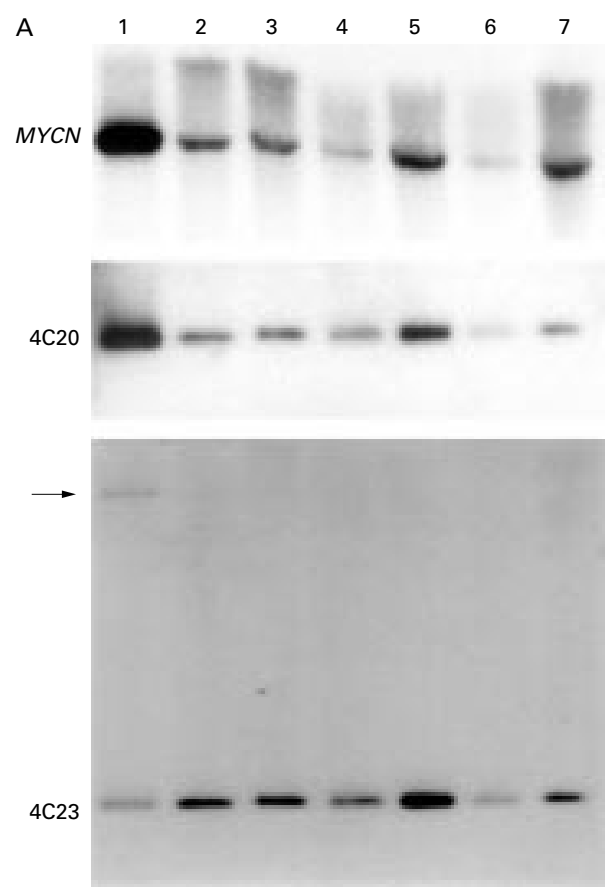

B

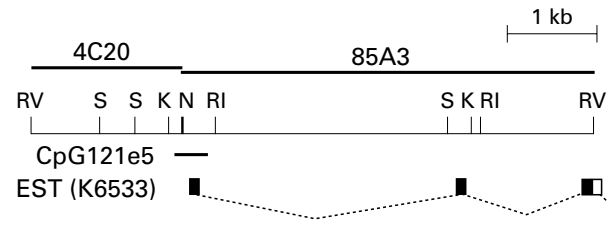

Figure 2 Analysis of NAG amplification in PNET/MB. (A) Representative Southern hybridisation showing coamplification of $N A G$ (4C20) with MYCN in the supratentorial PNET No 21; $7.5 \mu \mathrm{g}$ of DNA were digested with NotI/EcoRV electrophoresed and hybridised with probes for MYCN, 4C20 (5' CpG island of NAG) and $4 C 23$ as

described in Materials and methods. Lanes 1 and 2 represent the supratentorial PNETs No 21 (lane 1) and No 23 (lane 2), lanes 3-6 are the medulloblastomas No 15 (lane 3), No 3 (lane 4), No 18 (lane 5), and No 1 (lane 6). Lane 7 corresponds to normal PBL DNA from a newborn infant. While MYCN and 4C20 are amplified in tumour No 21 (lane 1), no amplification of 4C23 was detected in this sample. The closed arrow points to an additional band in sample No 21 possibly because of a rearrangement at this locus. (B) Restriction map of 4C20 and the adjacent NotI/EcoRV clone 85A3. Shown is an alignment of the restriction map of clone $4 C 20$ and the adjacent $3^{\prime}$ NotI/EcoRV clone $85 A 3$ with the sequence of the CpG island clone $121 e 5$ and EST $k 6533$. While $4 C 20$ represents part of the 5' CpG island of the novel NAG gene, EST $k 6533$ represents the first three putative exons of that gene.

Both clones mapped to the identical location between markers D2S131 and D2S312, a region on $2 \mathrm{p} 24.3$ coinciding with the $M Y C N$ region. ${ }^{36}$ Southern blot experiments showed that all tumours with $N A G$ amplification also had amplification of $M Y C N$. This suggested that both amplified fragments are part of the $M Y C N$ amplicon. Interestingly, MYCN was shown previously to be amplified in medulloblastomas only rarely. ${ }^{37}$

4C20 REPRESENTS A NOVEL GENE WITHIN THE MYCN AMPLICON

While 4C23 showed no homology to any known sequences in the database, 4C20 showed a high homology (100\% and $97 \%)$ over $40 \mathrm{bp}$ to two $\mathrm{CpG}$ island clones previously submitted to GenBank (clone cpg172a11 Accession No Z54901 and clone cpg121e5 Accession No Z54487) (fig 2B). These two clones had 52 bp homology with an EST derived from fetal heart (k6533, Accession No AA247962). EST clone k6533 was identified recently as part of $N A G$ (neuroblastoma amplified gene), which is amplified and overexpressed in a subset of neuroblastomas with MYCN amplification. ${ }^{16}$ Using a $170 \mathrm{bp}$ fragment from this EST as a probe for Southern hybridisation, we detected amplification of the same sized fragment as clone 4C20, indicating the homology of both fragments. In addition we obtained the NotI/EcoRV fragment $3^{\prime}$ of 4C20 from our NotI-EcoRV library by filter hybridisation using the PCR product from EST k6533 as a probe. Sequence analysis of this clone, $85 \mathrm{~A} 3$, and $\mathrm{k} 6533$ showed that these clones overlapped by $144 \mathrm{bp}$. Nucleotides 1-52 of k6533 are located immediately 3' of the NotI site, while bp 53-105 and 106-144 are located approximately $2.8 \mathrm{~kb}$ and $5 \mathrm{~kb}$ downstream of the NotI site respectively (fig $2 \mathrm{~B}$ ).

To determine the tissue distribution and the approximate transcript size of $N A G$, we used the k6533 probe to hybridise multi-tissue northern blots. Our results suggest a $N A G$ transcript size of $7 \mathrm{~kb}$, which is widely expressed in normal tissues, with highest levels in heart $(\mathrm{H})$ and skeletal muscle $(\mathrm{Sm})$ followed by brain $(\mathrm{Br})$, pancreas $(\mathrm{Pa})$, placenta $(\mathrm{Pl})$, and kidney (Ki). Little or no expression was observed in liver (Li), small intestine, and thymus (fig 3A, data for small intestine and thymus not shown).

Expression of $N A G$ was tested in two medulloblastoma samples (Nos 5 and 8). Since the amounts of RNA were insufficient for northern hybridisation a semiquantitative RTPCR assay was used. Expression levels of $N A G$ were compared to those of the housekeeping gene GPI. RNA from normal cerebella, PBL, adult brain, and five medulloblastoma cell lines were used as controls (fig 3B). Intensities of RT-PCR products were scanned using a phosphorimager. Relative levels of $N A G$ expression (compared to GPI) were determined for each sample (fig 3C). The relative expression in the cell lines ranged from 0.58 (lane $6=\mathrm{MHH}-$ PNET-5) to 1.56 (lane $4=$ MHH-MED-1) indicating the variability of this experiment. Relative expression levels in the patient samples ranged from 0.54 (lane $13=$ normal cerebellum 9609P325) to 1.36 (lane $9=\mathrm{MB}$ No 8). Only the adult brain sample (lane 3) showed a relatively low ratio, indicating either a reduced expression of $N A G$ in normal adult cerebral cortex compared to childhood cerebellum or an increased GPI expression. Tumour No 5, which showed the highest amplification levels of NAG by RLGS and by Southern analysis, did not show overexpression of the gene.

NAG MAPS 400 KB TELOMERIC TO MYCN

Since $N A G$ mapped to chromosome 2p24 and was coamplified with $M Y C N$ in PNET/MB samples, we hypothesised that $N A G$ might be in close physical proximity to $M Y C N$. To test 


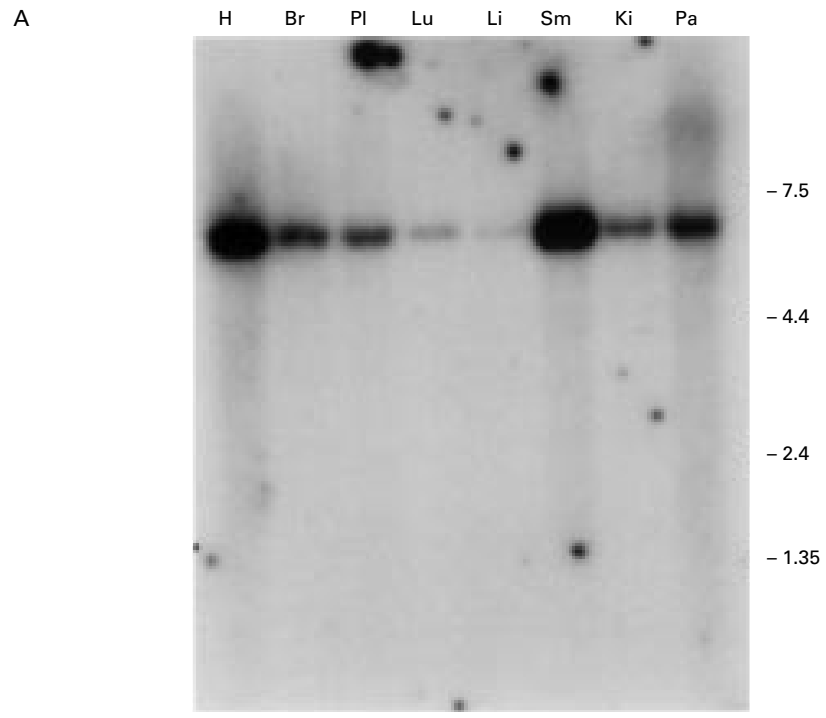

B
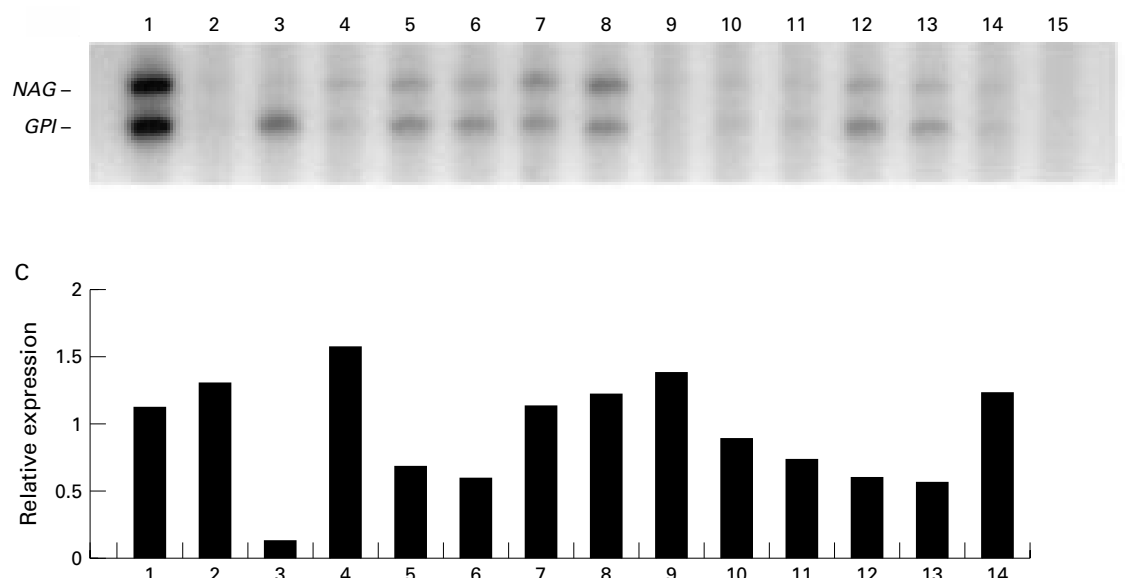

Figure 3 Expression analysis of NAG in medulloblastomas. (A) Multiple tissue northern blot hybridised with a probe derived from EST k6533, located 5' in the NAG gene. Hybridisation of $k 6533$ to poly $(A)+$ generated RNA shows a near ubiquitous expression of NAG. Highest expression levels are seen in heart $(H)$ and skeletal muscle (Sm) followed by pancreas (Pa), brain (Br), placenta (Pl), and kidney (Ki). The transcript size is about $7 \mathrm{~kb}$. (B) Autoradiography of RT-PCR products of NAG (210 bp) and GPI (178 bp) using RNA from control samples: PBL (lane 2), adult brain (lane 3), normal cerebella 9703P309 (lane 10), 9609P322 (lane 11), 9609P315 (lane 12), 9609P325 (lane 13), and 9704P318 (lane 14); medulloblastoma cell lines: MHH-MED-1 (lane 4), Daoy (lane 5), MHH-PNET-5 (lane 6), D341 Med (lane 7), D283 Med (lane 8); as well as medulloblastoma samples No 21 (lane 1) and No 8 (lane 9). Lane 15 is the negative control. (C) Relative expression of NAG compared to GPI in medulloblastoma and control samples based on intensity levels of the respective RT-PCR products. The order of sample numbers is as in (B).

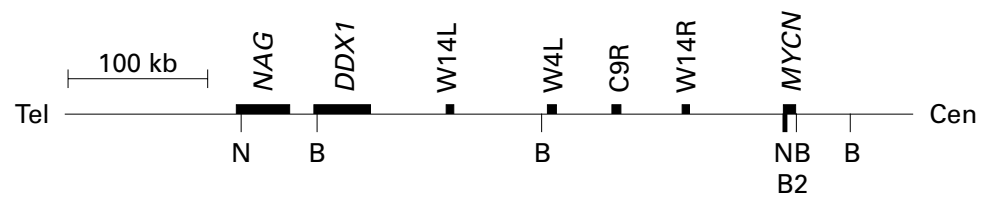

yCNL-12

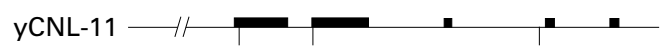

yWNL-14
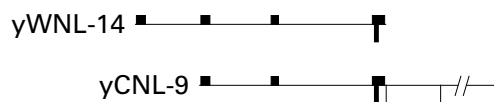

Figure 4 YAC map of the normal MYCN locus in 2p24. Physical map of MYCN, $D D X 1$, and NAG. The restriction and probe maps are based on a previous study. ${ }^{30}$ NotI $(N)$ and BssHII (B) restriction sites are shown as vertical lines. The probes W14L,W4L, $C 9 R$, and $W 14 R$ were derived from the left end (5') of YACs yWNL-14 and $y W N L-4$ and from the right end (3') of $y W N L-4$ and $y C N L-9$, respectively. TEL, telomeric; $C E N$, centromeric. this hypothesis we localised $N A G$ on a YAC contig spanning the $M Y C N$ locus. ${ }^{13}$

Four NotI sites are present in the contig; these sites are located within the $\mathrm{CpG}$ island of $M Y C N, 400 \mathrm{~kb}$ telomeric (5' of $M Y C N$ ), 600 $\mathrm{kb}$ and $750 \mathrm{~kb}$ centromeric of MYCN. We mapped EST k6533 by PCR and by Southern hybridisation to YACs yCNL-11 and yCNL-12 suggesting that $N A G$ is associated with the telomeric NotI site approximately 400 $\mathrm{kb} 5^{\prime}$ of $M Y C N$ (fig 4).

\section{Discussion}

We used RLGS to scan the genomes of medulloblastomas and supratentorial PNETs for DNA amplification. Our data indicate a slightly higher frequency of DNA amplification, but in general confirm the findings of other investigators that DNA amplification is a rare event in these tumours. ${ }^{9}{ }^{34} 35$ Even though we detected 
enhanced DNA fragments in up to seven of 25 tumours, there is very little overlap of amplified DNA sequences between tumours and some fragments are uniquely enhanced in only one tumour. This supports the notion that very few genes are consistently amplified in PNET/MB. The DNA fragments $4 \mathrm{C} 20,4 \mathrm{~F} 62,3 \mathrm{C} 15$, and $5 \mathrm{~B} 35$ were found enhanced in more than one tumour and may thus represent more commonly amplified genes. For example, 4C20 was amplified in three of the 25 analysed tumours and corresponds to the $5^{\prime} \mathrm{CpG}$ island of $N A G$. Our finding of a higher frequency in single locus amplifications compared to published data has to be validated by the characterisation of all amplified DNA sequences or genes detected by RLGS.

The gene that is thought to be the most commonly amplified gene in medulloblastomas, $M Y C,{ }^{7}$ was not amplified in any of the primary tumours when studied by RLGS. Southern analysis of eight of these tumours did not detect any MYC amplification. This finding allows only two interpretations: (1) $M Y C$ amplification is present in our samples, but does not involve the $5^{\prime} \mathrm{CpG}$ islands and therefore does not cause an enhancement of fragment 3B35, (2) the extent of MYC amplification in medulloblastomas has been overestimated and may need re-evaluation in a large number of tumours. The DNA fragment 4C3, amplified in tumour No 21, is located on chromosome 8 and also amplified in D341 Med, a cell line with a characteristic $M Y C$ amplification. It is possible that No 21 shows amplification of $M Y C$ in addition to amplification of $M Y C N$ and that $4 \mathrm{C} 3$ represents a gene within the $M Y C$ amplicon or part of the $M Y C$ gene itself.

The utility of the two dimensional gel electrophoresis method RLGS in the detection of known and novel amplified DNA sequences has been reported. ${ }^{18} 1939$ RLGS mixing gels allow for direct access to clones and DNA sequences. ${ }^{17}$ The majority of NotI restriction sites are located in $\mathrm{CpG}$ islands located mainly in the $5^{\prime}$ region of genes. ${ }^{40}$ Thus, RLGS profiles can be viewed as a display of $\mathrm{CpG}$ island or gene sequences. Support for this assumption is derived from our cloning of several known genes from RLGS profiles like $M Y C$, the oestrogen receptor, WIT-1, CDK6, and others. ${ }^{17} 2139-41$ Even though some of the enhanced $\mathrm{CpG}$ island fragments detected in the current study may map to the same amplicon (that is, 4C20, 4C23, 4F62, 4A5, 4A23, $3 \mathrm{C} 25$, and $4 \mathrm{~F} 53$ on chromosome 2 ), it is very likely that each fragment is associated with a different, possibly novel gene.

For example, CGH analysis of sample No 8 showed gains on chromosomes 4q16 and 5p15. RLGS analysis of the corresponding tumour detected two enhanced fragments, which may correspond to an amplicon recently described on chromosome 5p15.3. ${ }^{33}$ Furthermore, the study by Reardon $e t a l^{33}$ describes an additional amplicon on 11q22.3, which may well be represented by one or more of the enhanced fragments of our study, which have not been cloned at this time. To date no candidate oncogene has been described on $11 \mathrm{q} 22.3$ or $5 \mathrm{p} 15$.

The EST clone k6533, identified in our study, is part of a previously described gene, $N A G$, or neuroblastoma amplified gene. ${ }^{16} N A G$ was identified in a two dimensional gel electrophoresis analysis of neuroblastomas using a slightly different approach when compared to ours. While our enzyme combination is Not $\mathrm{I} /$ EcoRV/HinfI, Wimmer et al ${ }^{16}$ used NotI/EcoRV/ $D p n I I$. The DNA fragments therefore have different sizes and migrate to different locations on the profile. The fragment $4 \mathrm{C} 20$ is $355 \mathrm{bp}$ and $4 \mathrm{C} 23$ is slightly smaller, while NBA-2B of Wimmer et $a l^{16}$ is $455 \mathrm{bp}$ in size and NBA-2A is $139 \mathrm{bp}$. While the homology between NBA-2B and $4 \mathrm{C} 20$ is certain owing to the homology to the associated $\mathrm{CpG}$ island, we cannot be sure that $4 \mathrm{C} 23$ corresponds to NBA-2A. Secondly, these authors found a cDNA for the $N A G$ gene of $4.5 \mathrm{~kb}$, while our multi-tissue northern analysis shows a transcript size of approximately $7 \mathrm{~kb}$ (fig 3A). One explanation for the discrepancy in transcript size is that our probe (located in the 5' end of NAG) detects an alternative transcript of $N A G$ that was missed by using a probe located closer to the 3 ' end.

$N A G$ was previously localised telomeric to $M Y C N$ by double colour FISH. ${ }^{16}$ We have determined the exact location of $N A G$ on a YAC contig of the MYCN locus on chromosome 2p24. NAG maps to the telomeric Not I site in the contig approximately $400 \mathrm{~kb} 5^{\prime}$ of the NotI site associated with $M Y C N$. Since we did not detect any $N A G$ overexpression in the tested PNET/MB, the gene is most likely a coamplified gene in the MYCN amplicon. Several other genes have been reported to be coamplified with $M Y C N$ including ornithine decarboxylase $(O D C)$, ribonucleotide reductase (RRM2), syndecan-1 (SDC), propiomelanocortin (POMC), and DEAD box protein $(D D X 1) \cdot{ }^{42}$ Of these, only $D D X 1$ has been shown to be coamplified with $M Y C N$ in more than a few tumours.

Neither $D D X 1$ nor $N A G$ have been found amplified in the absence of $M Y C N$ amplification. Kuroda et al ${ }^{30}$ mapped $D D X 1340 \mathrm{~kb} 5^{\prime}$ of $M Y C N$ and therefore approximately $60 \mathrm{~kb} 3^{\prime}$ of the $N A G$ locus. Neither gene lies within the $130 \mathrm{~kb}$ core domain for MYCN amplification, defined in neuroblastomas. ${ }^{43}$ Furthermore, Reiter and Brodeur ${ }^{44}$ have shown that $M Y C N$ is the only consistently amplified and overexpressed gene in neuroblastomas.

In conclusion, we evaluated the frequency of gene amplifications in medulloblastomas and other PNETs of the CNS. The gene NAG, previously found amplified and overexpressed in neuroblastomas, is also amplified in a subset of PNET/MB but always coamplified with $M Y C N$. Therefore we suggest renaming it as "N-MYC coamplified gene" to stress the importance of $M Y C N$.

We would like to thank Dr T Pietsch (University of Bonn) for the MHH-MED-1 and MHH-PNET-5 cell lines, Fred Wright for his critical evaluation of part of the data, and Julie Eisel, Yue-Zhong Wu, and Suzanne Brady for expert technical assistance. The help of Lori McLoughlin (CHTN, Children's Hospiance. The help of Lori McLoughlin (CHTN, Children's Hospi-
tal, Columbus, OH) in obtaining tissue specimen is greatly tal, Columbus, OH) in obtaining tissue specimen is greatly
appreciated. This work was supported in part by the Children's 
Hospital Research Foundation grant No 216398, by grant No 216498 from Ladies Auxiliary of the Veterans of Foreign Wars (MSO), by T32 CA09338-20 Oncology Training Grant from the National Cancer Institute (DJS), by the National Cance Institute, Bethesda, MD, grant P30 CA16058, and the Bremer Foundation (CP). MCF was supported sequentially by a fellowship of the Dr Mildred Scheel Stiftung für Krebsforschung and by a fellowship of the Bauerstiftung im Stifterverband für die Deutsche Wissenschaft.

1 Caputy AJ, McCullough DC, Manz HJ, Patterson K, Hammock MK. A review of the factors influencing the prognosis of medulloblastoma. The importance of cell differentiation. F Neurosurg 1987;66:80-7

2 Harisiadis L, Chang CH. Medulloblastoma in children: a correlation between staging and results of treatment. Int $\mathcal{F}$ Radiat Oncol Biol Phys 1977;2:833-41.

3 Jenkin D, Goddard K, Armstrong D, et al. Posterior fossa medulloblastoma in childhood: treatment results and a proposal for a new staging system. Int 7 Radiat Oncol Biol Phys 1990;19:265-74

4 Duffner PK, Cohen ME, Myers MH, Heise HW. Survival of children with brain tumors: SEER Program, 1973-1980. children with brain tumors:

Neurology 1986;36:597-601.
5 Burnett ME, White EC, Sih S, von Haken MS, Cogen PH. Chromosome arm $17 \mathrm{p}$ deletion analysis reveals molecular genromosome arm $17 \mathrm{p}$ deletion analysis reveals molecular genetic heterogeneity in supratentorial and infratentorial primitive neuroectodermal tumors of the cen

6 Biegel JA, Janss AJ, Raffel C, et al. Prognostic significance of chromosome $17 \mathrm{p}$ deletions in childhood primitive neuroectodermal tumors (medulloblastomas) of the centra nervous system. Clin Cancer Res 1997;3:473-8.

7 Scheurlen WG, Schwabe GC, Joos S, Mollenhauer J, Sorensen N, Kuhl J. Molecular analysis of childhood primitive neuroectodermal tumors defines markers associated with poor outcome. $\mathcal{F}$ Clin Oncol 1998;16:2478-85.

8 Maris JM, Matthay KK. Molecular biology of neuroblastoma. F Clin Oncol 1999;17:2264.

9 Fuller GN, Bigner SH. Amplified cellular oncogenes in neoplasms of the human central nervous system. Mutat Res 1992;276:299-306.

10 Garson JA, Pemberton LF, Sheppard PW, Varndell IM Coakham HB, Kemshead JT. N-myc gene expression and
oncoprotein characterisation in medulloblastoma. $B r f$ oncoprotein characteris

11 Nisen PD, Zimmerman KA, Cotter SV, Gilbert F, Alt FW, Enhanced expression of the N-myc gene in Wilms' tumors. Cancer Res 1986;46:6217-22.

12 Schwab M. Amplification of oncogenes in human cancer cells. Bioessays 1998;20:473-9.

13 Schneider SS, Hiemstra JL, Zehnbauer BA, et al. Isolation and structural analysis of a 1.2 -megabase $\mathrm{N}$-myc amplicon from a human neuroblastoma. Mol Cell Biol 1992;12:5563 70 .

14 Akiyama K, Kanda N, Yamada M, Tadokoro K, Matsunaga T, Nishi Y. Megabase-scale analysis of the origin of N-myc amplicons in human neuroblastomas. Nucleic Acids Res 1994;22:187-93.

15 Squire JA, Thorner PS, Weitzman S, et al. Co-amplification of MYCN and a DEAD box gene (DDX1) in primary neuroblastoma. Oncogene 1995;10:1417-22.

16 Wimmer K, Zhu XX, Lamb BJ, et al. Co-amplification of a novel gene, NAG, with the N-myc gene in neuroblastoma. novel gene, NAG, with the

17 Smiraglia DJ, Frühwald MC, Costello JF, et al. A new tool for the rapid cloning of amplified and hypermethylated human DNA sequences from RLGS gels. Genomic 1999;58:254-262

18 Hirotsune S, Hatada I, Komatsubara $\mathrm{H}$, et al. New approach for detection of amplification in cancer DNA using restriction landmark genomic scanning. Cancer Res 1992;52 3642-7.

19 Curtis LJ, Li Y, Gerbault-Seureau M, et al. Amplification of DNA sequences from chromosome 19q13.1 in human pancreatic cell lines. Genomics 1998;53:42-55.

20 Pietsch T, Scharmann T, Fonatsch C, et al. Characterization of five new cell lines derived from human primitive neuroectodermal tumors of the central nervous system. Cancer Res 1994;54:3278-87.

21 Plass C, Shibata H, Kalcheva I, et al. Identification of Grf1 on mouse chromosome 9 as an imprinted gene by RLGSM. Nat Genet 1996;14:106-9.
22 Yoshikawa $\mathrm{H}$, de la Monte S, Nagai H, Wands JR, Matsubara K, Fujiyama A. Chromosomal assignment of human genomic NotI restriction fragments in a two-dimensional electrophoresis profile. Genomics 1996;31:28-35.

23 Plass C, Weichenhan D, Catanese J, et al. An arrayed human Not I-EcoRV boundary library as a tool for RLGS spot analysis. DNA Res 1997;4:253-5.

24 Miano JM, Krahe R, Garcia E, Elliott JM, Olson EN. Expression, genomic structure and high resolution mapping to $19 \mathrm{p} 13.2$ of the human smooth muscle cell calponin gene. Gene 1997;197:215-24

25 Hudson TJ, Stein LD, Gerety SS, et al. An STS-based map of the human genome. Science 1995;270:1945-54

26 Gyapay G, Schmitt K, Fizames C, et al. A radiation hybrid map of the human genome. Hum Mol Genet 1996;5:339-

27 Stewart EA, McKusick KB, Aggarwal A, et al. An STS-based radiation hybrid map of the human genome. Genome Res 1997;7:422-33.

28 Miano JM, Garcia E, Krahe R. High resolution radiation hybrid (RH) mapping of human smooth muscle-restricted genes. In: Baker AH, ed. Molecular biology of vascular diseases. Methods in Molecular Medicine Series. New York: Humana Press, 1999:25-35.

29 Kallioniemi A, Kallioniemi OP, Sudar D, et al. Comparative genomic hybridization for molecular cytogenetic analysis of solid tumors. Science 1992;258:818-21.

30 Kuroda H, White PS, Sulman EP, et al. Physical mapping of the DDX1 gene to $340 \mathrm{~kb} 5$ ' of MYCN. Oncogene 1996;13: 1561-5.

31 Miwa W, Yashima K, Sekine T, Sekiya T. Demethylation of a repetitive DNA sequence in human cancers. Electrophoresis 1995; 16:227-32.

32 Thoraval D, Asakawa J, Wimmer K, et al. Demethylation of repetitive DNA sequences in neuroblastoma. Genes Chrom Cancer 1996;17:234-44.

33 Reardon DA, Michalkiewicz E, Boyett JM, et al. Extensive genomic abnormalities in childhood medulloblastoma by comparative genomic hybridization. Cancer Res 1997;57: 4042-7.

34 Bigner SH, Friedman HS, Vogelstein B, Oakes WJ, Bigner $\mathrm{DD}$. Amplification of the c-myc gene in human medulloblastoma cell lines and xenografts. Cancer Res 1990;50: 2347-50.

35 Bruggers CS, Tai KF, Murdock T, et al. Expression of the c-Myc protein in childhood medulloblastoma. 7 Pediatr Hematol Oncol 1998;20:18-25.

36 Deloukas P, Schuler GD, Gyapay G, et al. A physical map of 30,000 human genes. Science 1998;282:744-6.

37 Rouah E, Wilson DR, Armstrong DL, Darlington GJ. $\mathrm{N}$-myc amplification and neuronal differentiation in human primitive neuroectodermal tumors of the central nervous system. Cancer Res 1989;49:1797-801.

38 Wasson JC, Saylors RLD, Zeltzer P, et al. Oncogene amplification in pediatric brain tumors. Cancer Res 1990;50:298790 .

39 Costello JF, Plass C, Arap W, et al. Cyclin-dependent kinase 6 (CDK6) amplification in human gliomas identified using two-dimensional separation of genomic DNA. Cancer Res 1997;57:1250-4

40 Costello JF, Frühwald MC, Smiraglia DJ, et al. Aberrant $\mathrm{CpG}$ island methylation has non-random and tumor type specific patterns. Nat Genet 2000;25:132-8.

41 Plass C, Yu F, Yu L, et al. Restriction landmark genome scanning for aberrant methylation in primary refractory and relapsed acute myeloid leukemia; involvement of the WIT-1 gene. Oncogene 1999;18:3159-65.

42 George RE, Kenyon RM, McGuckin AG, Malcolm AJ, Pearson AD, Lunec J. Investigation of co-amplification of the candidate genes ornithine decarboxylase, ribonucleotide reductase, syndecan-1 and a DEAD box gene, DDX1, with N-myc in neuroblastoma. United Kingdom Children's Cancer Study Group. Oncogene 1996;12:15837.

43 Reiter JL, Brodeur GM. High-resolution mapping of a 130 -kb core region of the MYCN amplicon in neuroblastomas. Genomics 1996;32:97-103.

44 Reiter JL, Brodeur GM. MYCN is the only highly expressed gene from the core amplified domain in human neuroblastomas. Genes Chrom Cancer 1998;23:134-40. 